\title{
Descripcion del sincraneo de Ameiva Ameiva (Linnaeus) (Squamata, Teiidae)
}

\author{
María Esther Tedesco ${ }^{1}$ \\ Lígia Krause ${ }^{2}$ \\ Blanca Beatríz Alvarez ${ }^{1}$
}

\begin{abstract}
Description of the syncranium of Ameiva Ameiva (Linnaeus) (Squamata, Teiidae). An osteological description of the syncranium and hyoid apparatus of Ameiva ameiva (Linnaeus, 1758) is presented. By comparison between males and females significant intersexual differences were found in relation to: skull size, frontal, parietal and basisphenoid bones, interpterygoid opening and teeth number and morphology. Absence of pterygoid teeth in A. ameiva is verified out. The specimens used for this study came from Chaco and Formosa provinces (Argentina).

KEY WORDS. Squamata, Teiidae, Ameiva ameiva, Neotropical region, skull
\end{abstract}

El género Ameiva (Meyer, 1795) se halla distribuido por el continente americano, desde Méjico e Islas del Caribe (ECHTERNACHT 1971), hasta el norte de Argentina (CEI 1993).

Comprende 35 especies, la mayoría de las cuales habitan países de América Central y solo 6 se distribuyen en América del Sur. De éstas últimas A. ameiva (Linnaeus, 1758) posee un amplio rango de distribución geográfica, que se extiende desde las sabanas de América Central (ECHTERNACHT 1971), hasta los bosques subtropicales húmedos en Argentina.

Vive en simpatría con Cnemidophorus ocellifer (Spix, 1825) y Teius teyou (Daudin, 1802) en ambientes de pastizales y bosques subxerófilos del norte de Argentina, Paraguay occidental y oriental y Brasil nororiental.

Con el propósito de aportar datos significativos que contribuyan a una mejor comprensión del status taxonómico de $A$. ameiva, se encaró el estudio osteológico descriptivo del sincráneo de este taxa.

Pocos son los antecedentes que existen sobre estudios osteológicos craneales en teidos sudamericanos, entre ellos REESE(1923) Tupinambis nigropunctatus Spix, 1825 [actualmente T. teguixin, Linnaeus, 1758]; BARBERENA et al. (1970) Tupinambis teguixin (Linnaeus, 1758) [actualmente T. merianae (Duméril \& Bibron, 1839)]; Alvarez et al. (1987) Teius teyou (Daudin, 1802)

Sobre el mismo tema pero en teidos centro y norteamericanos FISHER \& TANNER (1970) estudiaron Ameiva undulata parva Barbour \& Noble, 1915 y Cnemidophorus tigris septentrio-nalis Burger, 1950.

1) Departamento de Biología, Faculdad de Ciencias Exactas y Naturales, Universidad Nacional del Nordeste. 9 de julio 1449, 3400 Corrientes, Argentina.

2) Departamento de Zoologia, Instituto de Biociências, Universidade Federal do Rio Grande do Sul. Avenida Paulo Gama, Prédio 12106, 90046-900 Porto Alegre, Rio Grande do Sul, Brasil. 


\section{MATERIALES Y METODOS}

Fueron utilizados 20 especímenes de $A$. ameiva, 10 machos y 10 hembras.

El material procede de diferentes localidades de las provincias de Chaco y Formosa (Argentina) y están depositados en la Colección Herpetológica de la Universidad Nacional del Nordeste-Corrientes. (UNNEC).

Debido a la fragilidad de las estructuras óseas, los cráneos fueron preparados manualmente.

Con el objeto de diferenciar los elementos óseos y cartilaginosos se realizó diafanización y tinción diferencial, mediante la técnica de WASSERSUG (1976).

Para verificar diferencias intersexuales se analizaron 12 variables métricas, las que fueron comparadas mediante el Test t donde $\alpha=0,05$. Además se realizó un análisis de componentes principales sobre la matriz de correlación (coeficiente de correlación lineal de Pearson) (Tab. I, Fig. 1).

Tabla I. Promedios (X), desvio standard (DS), Test $t(t)$ y coeficiente de Pearson (p) para las variables métricas $(\mathrm{N}=12)$ entre machos y hembras.

\begin{tabular}{|c|c|c|c|c|c|c|}
\hline \multirow{2}{*}{ Variables } & \multicolumn{2}{|c|}{ Promedios } & \multicolumn{2}{|c|}{ Desvio standard } & \multirow{2}{*}{ Test t } & \multirow{2}{*}{$\begin{array}{c}\text { Coeficiente de } \\
\text { Pearson }\end{array}$} \\
\hline & Machos & Hembras & Machos & Hembras & & \\
\hline Longitud total del cráneo & 3,775 & 3,121 & 0,32 & 0,17 & 5,64 & 0,0001 \\
\hline Ancho del cráneo & 1,928 & 1,460 & 0,20 & 0,74 & 6,78 & 0,0001 \\
\hline Altura del cráneo & 1,311 & 1,045 & 0,15 & 0,09 & 4,66 & 0,0002 \\
\hline Longitud de las narinas & 0,640 & 0,590 & 0,06 & 0,04 & 0,09 & 0,0512 \\
\hline Altura de las narinas & 0,377 & 0,351 & 0,04 & 0,03 & 1,84 & 0,0820 \\
\hline Longitud del cuerpo del parietal & 1,673 & 1,300 & 0,18 & 0,14 & 5,07 & 0,0001 \\
\hline Ancho del cuerpo del parietal & 0,371 & 0,351 & 0,04 & 0,03 & 1,37 & 0,1900 \\
\hline Longitud abertura orbitaria & 0,730 & 0,540 & 0,10 & 0,07 & 4,78 & 0,0001 \\
\hline Altura de la abertura orbitaria & 0,898 & 0,784 & 0,04 & 0,05 & 5,59 & 0,0001 \\
\hline Longitud hocico & 1,112 & 1,010 & 0,06 & 0,07 & 3,58 & 0,0021 \\
\hline Ancho del premaxilar & 0,958 & 0,799 & 0,08 & 0,07 & 4,72 & 0,0002 \\
\hline Distancia narina-órbita & 0,975 & 0,732 & 0,13 & 0,08 & 4,89 & 0,0001 \\
\hline
\end{tabular}

Se analizó la morfología e implantación de los dientes premaxilares, maxilares y mandibulares. Se halló la fórmula dentaria utilizándose la moda como el valor más representativo del conjunto (Tab. II).

Tabla II. Número mínimo y máximo de dientes de la arcada dentaria de $A$. ameiva. (Pmx) Premaxilar, (Mx) maxilar; (D) dentario, (U) unicuspidal, (B) bicuspidal; (T) tricuspidal.

\begin{tabular}{|c|c|c|c|c|c|c|c|}
\hline \multirow[b]{2}{*}{ Sexo } & \multirow{2}{*}{ Premaxilar } & \multicolumn{3}{|c|}{ Maxilar } & \multicolumn{3}{|c|}{ Dentario } \\
\hline & & Unicuspidal & Bicuspidal & Tricuspidal & Unicuspidal & Bicuspidal & Tricuspidal \\
\hline Machos & $9-11$ & $2-5$ & $7-12$ & $6-11$ & $3-4$ & $5-10$ & $9-11$ \\
\hline Hembras & $8-11$ & $1-6$ & $11-20$ & $2-6$ & $2-9$ & $11-20$ & $2-7$ \\
\hline
\end{tabular}

Las medidas de las variables fueron obtenidas de la siguiente manera:

- Longitud total del cráneo (LC): desde el extremo anterior del hocico hasta el extremo posterior del cóndilo occipital.

- Ancho del cráneo (AC): a nivel de los huesos cuadrados por ser la región más ancha. 


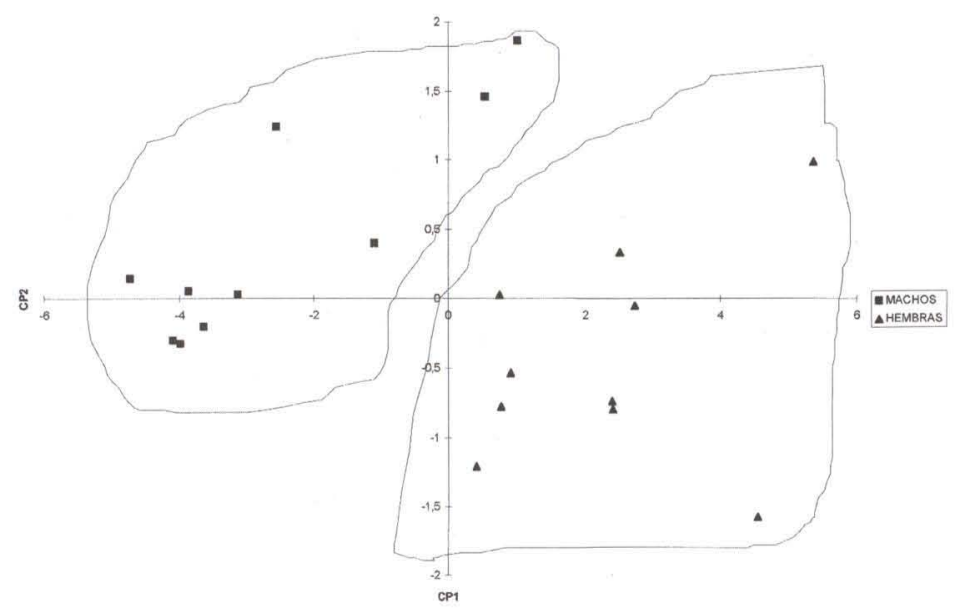

Fig. 1. Identificación del dimorfismo sexual a través de la técnica de componentes principales.

- Altura del cráneo (HC): a nivel de los procesos basipterigoides del basiesfenoides y dorsalmente sobre el parietal.

- Longitud de las narinas (LN): desde el borde posterior del proceso maxilar del premaxilar hasta la escotadura del hueso nasal anteriormente.

- Altura de las narinas (AN): desde la sutura del premaxilar con el maxilar hasta el proceso nasal del premaxilar dorsalmente.

- Longitud del cuerpo del parietal (LP): a nivel de la sutura anterior con el frontal hasta la sutura del parietal con el supraoccipital.

- Ancho del cuerpo del parietal (AP): distancia entre las dos proyecciones ánterolaterales del hueso donde se une el postfrontal-postorbital.

- Longitud abertura orbitaria (LAO): desde el hueso lagrimal al borde anterior del postfrontal-postorbital.

- Altura de la abertura orbitaria (HAO): a nivel del proceso ventral del yugal y el frontal dorsalmente.

- Longitud hocico (Lho): desde el borde anterior del premaxilar hasta el borde anterior de la órbita delimitado por el prefrontal.

- Ancho del premaxilar (Apm): a ambos lados de la sutura del premaxilar con el maxilar.

- Distancia narina-órbita (DNO): desde el borde posterior de las narinas al borde anterior de la órbita delimitado por el hueso prefrontal.

Los dibujos fueron realizados con microscopio estereoscópico Olympus SZH 10, con aumento de 4x el tamaño original del cráneo.

En la descripción del cráneo, se sigue la secuencia propuesta por GAVRILOV (1959) y BARBERENA et al. (1970).

La terminología utilizada es la propuesta por OELRICH (1956); BARBERENA et al. (1970) y SKINNER (1973).

La abreviatura utilizada corresponde a UNNEC - Universidad Nacional del Nordeste-Corrientes. 
Material estudiado: UNNEC: 01778, Nueva Pompeya, Chaco; 01779, Las Lomitas, Formosa; 01780, Nueva Pompeya, Chaco; 01781-01782, Fuerte Esperanza, Chaco; 01783, El Tayí, Formosa; 01784, Avia Terai, Chaco; 01785-01786, El Mistolar, Formosa; 01787, Puerto Tirol, Chaco, (10 machos); 01788, Comandante Fontana, Formosa; 01789-01790-01791, El Mistolar, Formosa; 01792-0179301794, Avia Terai, Chaco; 01795, Paraje Kolbar, Chaco; 01796-01797, Nueva Pompeya, Chaco, (10 hembras). Todos de Argentina.

\section{RESULTADOS}

En general el sincráneo de $A$. ameiva responde al patrón básico de la familia Teiidae, pudiéndose observar que la región del hocico representa aproximadamente un tercio de la longitud total del cráneo, y que adopta una forma alargada y aguzada, característica que lo diferencia de los demás teidos.

En su conjunto consta de 23 huesos pares y 6 impares. Cada rama mandibular consta de 6 huesos.

\section{Huesos marginales portadores de dientes}

\section{Premaxilar (Figs 2A,B, 3A)}

Elemento impar y más anterior del cráneo. Su forma es semejante a un ancla y es portador de dientes subpleurodontes. Presenta dos procesos: maxilar y nasal.

El proceso maxilar, articula con el proceso premaxilar del maxilar y con el extremo anterior del vómer. Forma el borde ánteroventral de la exonarina. Tanto en su cara dorsal como ventral, se abren forámenes que dan paso a las ramas mediales de la arteria subnarial y del nervio etmoidal (SKINNER 1973).

El proceso nasal largo, aguzado, penetra como cuña entre los nasales. Su cara dorsal es convexa y la ventral levemente cóncava, recorrida medialmente en toda su longitud por una cresta muy prominente por medio de la cual articula al septo maxilar. Sus bordes laterales delimitan el borde dorsal de la exonarina.

\section{Maxilar (Figs $2 A, B$ y $3 A$ )}

Hueso grande, forma la superficie lateral del hocico. Presenta tres procesos: premaxilar, nasal y posterior.

El proceso premaxilar bífido, su rama más corta de posición lateral, lleva dorsalmente una carilla articular plano-cóncava sobre la que se apoya la superficie ventral del proceso maxilar del premaxilar. La rama más larga, medial, articula con el borde medial del proceso maxilar del premaxilar, lateral y medialmente con el borde anterior y externo del septo maxilar y ventralmente con el proceso anterior del vómer. Dorsalmente es cóncavo y forma parte de la base de la fenestra exonarina. En su extremo posterior se abre el forámen alveolar superior para la arteria maxilar y el nervio alveolar superior (SKINNER 1973).

El proceso nasal forma la pared láterodorsal de la cápsula nasal y su borde anterior forma el borde posterior de la fenestra exonarina. Articula dorsalmente con el nasal y posteriormente con el prefrontal y lagrimal. Este proceso lleva una serie de forámenes, que transmiten ramas cutáneas del nervio etmoidal lateral y alveolar superior (OELRICH 1956; BARBERENA et al. 1970; SKINNER 1973). Internamente es recorrido por una cresta muy conspicua que nace en el piso de la placa palatal, y que divide a la cara interna en dos áreas. 



Fig. 2. Cráneo de $A$. ameiva, (A) vista dorsal y (B) vista ventral. (a.exn.) Abertura exonarina, (a.inf.) abertura infroorbitaria, (a.ipt.) abertura interpterigoidea, (bas.) basiesfenoides, (bocc.) basioccipital, (cuad.) cuadrado, (ecpt.) ectopterigoides, (esc.) escamoso, (f.exo.) fenestra exocoanal, (fr.) frontal, (f.supt.) fosa supra-temporal, (f.v.nas.) fenestra vómero nasal, (lagr.) lagrimal, (max.) maxilar, (nas.) nasal, (occ.) cóndilo occipital, (pal.) palatino, (par.) parietal, (pesf.) paraesfenoides, (pfr.) prefrontal, (pmax.) premaxilar, (pr.po.ex.) proceso paraoccipital del exoccipital, (pt.) pterifoides, (ptfpto.) postfrontal-postorbital, (socc.) supraoccipital, (supt.) supratemporal, (vom.) vómer, (yug.) yugal.

El proceso posterior situado por debajo de la órbita, articula con el lagrimal. Medialmente lleva un surco profundo en el cual se aloja el borde ventral de la rama anterior del yugal. Articula con el proceso transverso del yugal, con el transverso y el palatino.

La cara interna del hueso lleva la placa palatal, que se extiende desde el extremo anterior del proceso premaxilar hasta el extremo del proceso posterior.Soporta dorsalmente la cápsula nasal y ventralmente a los dientes subpleurodontes. Su borde interno forma el límite lateral de la fenestra vómeronasal y la fenestra exocoanal. 
La placa palatal está perforada por un túnel con un agujero alveolar que se abre a nivel del proceso posterior, por donde penetran el nervio alveolar superior y la arteria maxilar (SKINNER 1973).

Externamente el hueso lleva en el borde inferior una serie de orificios labiales por donde salen las ramas cutáneas del nervio alveolar superior y de la arteria maxilar (OELRICH 1956; SKINNER 1973).

\section{Región nasoetmoidal}

\section{Nasal (Figs 2A, 3A)}

Unidos en la línea media del cráneo, forman el techo de las fosas nasales. Dorsalmente es rugoso y convexo en su parte anterior. Lateralmente lleva una serie de forámenes para las ramas cutáneas del nervio etmoidal lateral (OELRICH 1956). Presenta tres procesos: anterior; maxilar y frontal.

Proceso anterior: largo, aguzado, articula con el proceso nasal del premaxilar.

Proceso maxilar: corto, en su borde lateral externo lleva un surco profundo que aloja al proceso nasal del maxilar. Posteriormente articula con el borde interno del prefrontal.

Entre ambos procesos hay una escotadura que delimita el borde posterior de la fenestra exonarina.

Proceso frontal (BARBERENA et al. 1970): largo, con los extremos romos, lleva medialmente un surco poco profundo para el proceso nasal del frontal y lateralmente una carilla articular para los procesos laterales del frontal.

\section{Septomaxilar (Fig. 3A)}

Situado ventral al nasal, de forma irregular, recubre al órgano de Jacobson y aloja la parte anterior de la cápsula olfatoria. Presenta tres procesos.

Proceso premaxilar: articula anterior y medialmente con el proceso premaxilar del maxilar. Lateralmente lleva una cresta.

Proceso posterior: corto, articula con el vómer y el maxilar.

Proceso dorsal: constituye el tabique nasal con el del lado opuesto. Articula dorsalmente con el proceso nasal del premaxilar.

\section{Vomer (Fig. 2B)}

Hueso alargado, forma la parte anterior del paladar, el borde medial de la fenestra vómeronasal y la fenestra exocoanal. Se hallan unidos a lo largo de toda su longitud por lo que no se observa el seno palatal medio. El extremo anterior aguzado penetra como cuña entre los procesos maxilares del premaxilar con quien articula; lateralmente articula con el borde medial del proceso premaxilar del maxilar; posteriormente con el proceso vomeriano del palatino al cual se le superpone. La región media del hueso presenta una expansión aliforme la que finaliza en un extremo posterior aguzado.

Presenta tres caras: dorsolateral, ventral y medial, esta última es perpendicular a la primera. La cara dorsolateral y la ventral se hallan divididas en su tercio anterior por una cresta transversal (SKINNER 1973), que se extiende en forma oblicua hacia la región posterior delimitando dos áreas, una pequeña anterior que soporta al órgano de Jacobson, y otra posterior que soporta al saco nasal. Inmediatamente 
por detrás de la cresta transversal hay un gran forámen a través del cual la rama medial del nervio palatino y la arteria entran al hueso (SKINNER 1973).
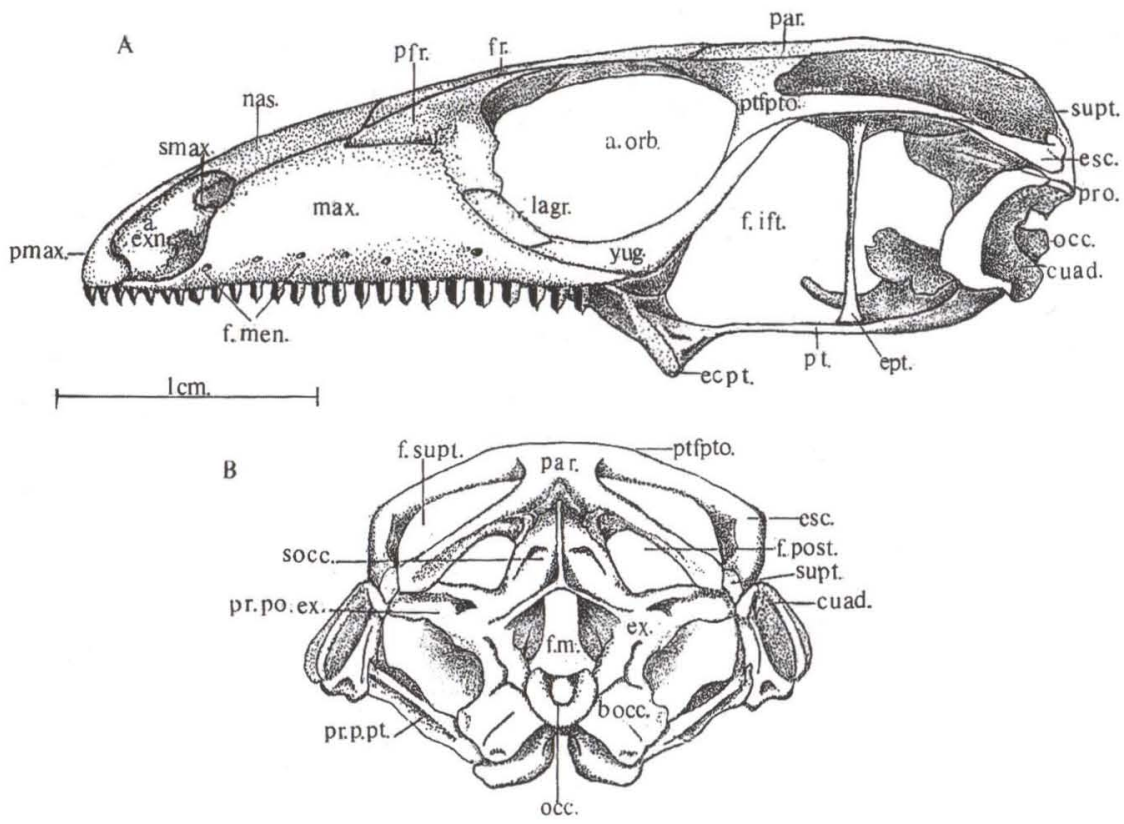

Fig. 3. Cráneo de A. ameiva, (3A) vista lateral y (3B) vista posterior occipital. (a.exn.) Abertura exonarina, (a.orb.) abertura orbitaria, (bocc.) basioccipital, (cuad.) cuadrado, (ecpt.) ectopterifoides, (ept.) epipterigoides, (esc.) escamoso, (ex.) exoccipital, (f.m.) forámen magnum, (f. ift.) fosa infratemporal, (f.men.) forámenes mentales, (f.post.) fosa postemporal, (f.supt.) fosa supra-temporal, (fr.) frontal, (lagr.) lagrimal, (max.) maxilar, (nas.) nasal, (occ.) cóndilo occipital, (par.) parietal, (pfr.) prefrontal, (pmax.) premaxilar, (pro.) proótico, (pr.p.pt.) proceso posterior del pterigoides, (pr.po.ex.-proceso paraoccipital del exoccipital, (pt.) pterigoides, (ptfpto.) postfrontal-postorbital, (smax.) septo maxilar, (socc.) supraoccipital, (supt.) supratemporal, (yug.) yugal.

\section{Palatino (Fig. 2B)}

Forma parte del piso de la órbita y de la región posterior de la cápsula nasal. Constituye gran parte del paladar junto con el vómer y pterigoides. Su borde anterior limita posteriormente la fenestra exocoanal y medialmente la fenestra suborbital. Presenta cuatro procesos: vomeriano, maxilar-ectopterigoides, prefrontal y pterigoides.

Proceso vomeriano: es medial. Se halla cubierto ventralmente por el extremo posterior del vómer. Este proceso se continúa hacia atrás formando la cresta palatina delimitando medialmente el surco palatino que aloja a la rama principal medial del plexo palatino VII. En la base de la cresta y a lo largo de toda su longitud hay una serie de forámenes por donde pasan ramas del nervio palatino a la membrana oral (SKINNER 1973). 
Proceso maxilar-ectopterigoides: situado lateralmente, articula con el proceso posterior del maxilar y con el ectopterigoides.

Proceso prefrontal: dirigido dorsalmente, ancho, de reborde espeso. Articula en toda su extensión con el proceso palatino del prefrontal. Forma una parte de la pared anterior de la órbita. Ventralmente delimita el borde posterior de la fenestra exocoanal.

Proceso pterigoides: es bífido. Medialmente lleva un pequeño surco para su articulación con el proceso palatino del pterigoides.

\section{Pterigoides (Figs 2B, 3A)}

Hueso grande, situado en la mitad posterior de la región palatal y separado medialmente de su opuesto por la vacuidad interpterigoidea. Dorsalmente, junto con el palatino y transverso, forma el piso de la órbita. Ventralmente constituye la parte posterior del paladar óseo. Este hueso carece de dientes. Anteriormente presenta dos procesos.

Proceso palatino: ancho, bifurcado. Su borde medial limita la vacuidad interpterigoidea.

Proceso transverso: es rectangular, penetra como cuña en el canal que presenta el proceso pterigoides del transverso.

Tanto el proceso palatino como el proceso transverso forman el borde posterior de la fenestra suborbitaria.

Proceso posterior: es aliforme. En la base del proceso hay una carilla articular plana sobre la que se apoya el proceso basipterigoides del basiesfenoides. Lateralmente articula con la superficie interna del hueso cuadrado. Dorsalmente lleva la fosa columelar, en la cual se inserta la extremidad ventral del epipterigoides.

\section{Ectopterigoides (Figs 2B, 3A)}

Hueso trirradiado, constituye el brazo entre el pterigoides y el maxilar. Forma parte del piso de la órbita y el borde lateral de la fenestra suborbitaria. Presenta tres procesos.

Proceso anterior: penetra como cuña entre el maxilar y el palatino. Lateralmente lleva una gran carilla articular para el proceso posterior del maxilar.

Proceso lateral externo: corto, penetra como cuña entre el maxilar y yugal con los cuales articula.

Proceso pterigoides: Presenta dos ramas en forma de horquilla, una ventral corta, espesa, en forma de tubérculo con el extremo distal redondeado. La otra, dorsal, más larga con dos apófisis, la medial interna es muy conspicua y aguzada. Entre ambas ramas hay un profundo canal que aloja al proceso transverso del pterigoides e interviene en la recepción del coronoides durante la aducción

\section{Epipterigoides (Fig. 3A)}

Hueso alargado, cilíndrico. Se distinguen en él dos extremos, uno dorsal que articula con la superficie lateral del proceso descendente del parietal y otro ventral que lleva en su extremidad distal un cóndilo aplanado que inserta en la fosa columelar del hueso pterigoides. 


\section{Región fronto-orbital}

\section{Frontal (Fig. 2A)}

Hueso, impar, alargado, comprimido en su parte media. Forma el borde dorsal de la órbita y el techo de la región anterior del cerebro.

Articula anteriormente con los procesos frontales del nasal y posteriormente con el parietal a través de una sutura dentada

Dorsalmente es rugoso y ventralmente liso, .con una concavidad anterior enmarcada por las crestas craneales que se prolongan hacia atrás formando un estrecho surco y hacia abajo formando los procesos descendentes (SKINNER 1973). El surco con la concavidad anterior forman el canal olfatorio que aloja a los pedúnculos olfatorios (BARBERENA et al. 1970; OELRICH 1956).

Anteriormente el hueso lleva tres apófisis, una central, alargada, aguzada, que penetra como cuña entre ambos nasales y dos laterales más cortas que articulan con los nasales y el borde interno del prefrontal.

\section{Prefrontal (Figs 2A, 3A)}

Forma el ángulo anterior de la órbita. Dorsalmente es convexo, levemente rugoso y lleva en su borde lateral una serie de forámenes. Presenta cuatro procesos.

Proceso anterior: redondeado, con una gran carilla articular para el proceso nasal del maxilar, medialmente articula con el borde lateral del nasal.

Proceso lateral: pequeño, representado por una lámina delgada y expandida. En ella se apoya el extremo anterior del lagrimal. La base del proceso forma el techo del agujero lagrimal.

Proceso palatino (BARBERENA et al. 1970; SKINNER 1973) es ancho, cóncavo; lateralmente lleva una pequeña apófisis que articula con el hueso lagrimal formando el ángulo ventral del agujero lagrimal. Ventralmente articula con el proceso prefrontal del palatino. Forma la pared anterior de la órbita, el borde medial del conducto lagrimal y delimita la fenestra órbitonasal.

Proceso frontal (SKINNER 1973): es alargado y comprimido lateralmente. Forma el borde anterior de la órbita. En la base del proceso se abre un forámen que transmite un brazo lateral del nervio oftálmico profundo que va a la órbita (SKINNER 1973).

\section{Postfrontal-postorbital (Figs 1A, B, 2A, B)}

Fusionado, tetrarradiado. Se distinguen en él tres procesos:

Proceso frontoparietal: orientado medialmente, presenta dos ramas, una anterior situada ventralmente, es larga y articula en su totalidad con el borde lateral del frontal. La posterior, es corta y penetra en la fosa lateral del parietal Este proceso forma el ángulo anterior de la fenestra supratemporal y el ángulo posterior de la órbita.

Proceso yugal (BARBERENA et al. 1970): situado ventralmente, es corto, triangular, su borde ventral lleva una gran carilla articular para el proceso temporal del yugal.

Proceso escamoso (BARBERENA et al. 1970): es delgado y aguzado, forma junto con el escamoso el borde externo de la fenestra supratemporal. 


\section{Lagrimal (Figs 2A, 3A)}

Hueso pequeño, de forma aproximadamente rectangular. Forma parte del borde ánteroventral de la órbita y el borde lateral del agujero lagrimal. Articula dorsalmente con el prefrontal, anteriormente con el proceso nasal del maxilar, medialmente con el borde láteroventral del proceso palatino del prefrontal.

Se distinguen en él dos caras: una lateral plana que articula ventralmente con el proceso posterior del maxilar y otra medial cóncava, que cierra lateralmente el agujero lagrimal. Posteriormente lleva el proceso yugal que se apoya sobre el proceso anterior del hueso yugal.

\section{Yugal (Figs 2A, B, 3A)}

Hueso alargado, curvado. Forma el borde ventral de la órbita. Presenta tres procesos.

Proceso anterior: ancho, aplanado; lateralmente articula con el proceso yugal del lagrimal. Ventralmente lleva un surco para la articulación del proceso posterior del maxilar; medialmente articula con el borde lateral del transverso.

Proceso posterior: es alargado y aguzado. Internamente lleva un surco en el que se apoya el borde ventral del proceso yugal del postfrontal-postorbital.

Proceso ventral: corto, articula con el proceso lateral del transverso y con el proceso posterior del maxilar. En él se abre el forámen para las ramas maxilares del trigémino (BARBERENA et al. 1970; SKINNER 1973), que emergen en la cara lateral del hueso a través de forámenes pequeños.

\section{Región esfeno-parietal-ótica}

\section{Parietal (Figs 2A, 3B)}

Hueso grande, impar, deprimido dorsoventralmente, forma el tercio posterior del techo del cráneo. La superficie dorsal muy rugosa, lleva dos crestas laterales que nacen en los procesos frontales y finalizan en el extremo posterior de los procesos parietales. Delimitan el borde interno de la fenestra supratemporal.

Articula anteriormente con el frontal a través de una sutura dentada, ventralmente con el supraoccipital. Presenta tres procesos.

Proceso frontal (BARBERENA et al. 1970): es corto, articula anteriormente con el frontal y lateralmente con el proceso frontoparietal del postfrontal-postorbital.

Proceso parietal (SKINNER 1973): situado en el ángulo posterior del hueso, es largo, y comprimido lateralmente. Articula lateral y ventralmente con el supratemporal. En la bifurcación de ambos procesos, en su parte media se abre el forámen de la fosa parietal que recibe al proceso cartilaginoso ascendente del supraoccipital (SKINNER 1973).

Proceso descendente: situado entre los procesos frontal y parietal; es triangular con el vértice dirigido ventralmente. En él se apoya el extremo dorsal del epipterigoides.

\section{Escamoso (Figs 2A,B, 3A,B)}

Hueso alargado con la extremidad anterior aguzada, la posterior en forma de gancho. Junto con el postfrontal-postorbital forman el borde externo de la fenestra supratemporal. Presenta tres procesos. 
Proceso anterior: largo, aguzado. Dorsalmente lleva un gran surco articular que aloja al proceso escamoso del postfrontal-postorbital.

Proceso dorsal: con forma de gancho. Articula con la cara externa del supratemporal. Forma el ángulo posterior de la fenestra supratemporal.

Proceso ventral: es corto y ancho. Articula al proceso posterior del supratemporal. Ventralmente posee una protuberancia que penetra en el agujero del hueso cuadrado.

\section{Basiesfenoides (Fig. 1B)}

Hueso impar, forma la parte anterior del piso de la cavidad craneal. Anterolateralmente presenta un par de procesos basipterigoides que articulan con los procesos cuadrados del pterigoides. Anterior y medialmente está unido a un hueso dérmico, impar, largo, aguzado en su extremo anterior, el paraesfenoides, que se extiende hasta el vértice de la cavidad interpterigoidea.

El proceso basipterigoides junto con el paraesfenoides delimitan una escotadura en cuyo centro se abre el forámen que corresponde a una de las ramas del canal vidiano por donde pasan la arteria y el nervio palatino.

En la cara dorsal hacia la parte posterior se encuentra la crista sellaris (BARBERENA et al. 1970) que la divide en dos áreas, una anterior cóncava y otra posterior. En la posterior se encuentran los forámenes que corresponden al canal abducente para el nervio abducente y lleva lateralmente dos pequeños procesos los cuales articulan con el basioccipital y proótico respectivamente. Dorsal y lateralmente la crista sellaris lleva los procesos alares que articulan con los procesos inferiores del proótico. Desde la crista sellaris desciende en forma perpendicular una pared cóncava, la dorsum sellaris. En ella hay un par de pequeños orificios que corresponden al canal abducente para el nervio abductor. Anterior a la dorsum sellaris hay un receso cóncavo, la sella turcica, que aloja al saco pituitario y lleva medialmente un par de forámenes grandes para el pasaje de la rama principal de la carótida interna.

La sella turcica, se halla enmarcada por la cresta lateral que nace en la base de los procesos alares y se extiende anteriormente hasta el proceso paraesfenoides. La cara lateral presenta una gran concavidad en la cual se abre el orificio posterior del canal vidiano por donde penetran la carótida interna y la rama palatina del nervio facial (SKINNER 1973). Anterior al canal vidiano hay una depresión para la vena yugular interna, limitada dorsal y anteriormente por la cresta supravenosa continuación de la proótica

\section{Supratemporal (Figs 3A, 3B)}

Hueso pequeño, situado en el ángulo posterior del cráneo. Presenta dos procesos.

Proceso anterior: es aguzado, dorsalmente lleva un profundo canal que aloja al borde ventral del proceso posterior del parietal. Limita una pequeña parte del borde posterior e interno de la fenestra supratemporal.

Proceso posterior: alargado con el extremo posterior dirigido ventralmente. Lateralmente lleva un profundo surco que recibe al proceso dorsal del escamoso con el cual articula, además de hacerlo con el parietal, exoccipital y cuadrado. 


\section{Cuadrado (Figs 2A,B, 3A,B)}

Hueso de forma auricular, situado en el ángulo pósterolateral del cráneo. Provee el área de articulación entre el cráneo y la mandíbula. Es estrecho en su extremidad dorsal y se ensancha hacia la ventral. Presenta dos caras, una ánteromedial y otra pósterolateral y dos superficies articulares, una dorsal representada por el cóndilo cefálico y otra ventral, el cóndilo mandibular.

La cara ánteromedial se halla dividida por un surco en dos áreas, una externa ancha, ligeramente convexa, con un reborde lateral espeso que se adelgaza en dirección al cóndilo mandibular. El área interna, es angosta y de superficie planocóncava con un reborde espeso.

La cara pósterolateral, presenta al igual que la cara ánteromedial dos áreas, una externa y otra interna, ambas separadas por la cresta posterior que se extiende desde el cóndilo cefálico hasta el mandibular ensanchándose a este nivel. El área externa es ancha, cóncava con un reborde muy conspicuo, la cresta timpánica (OELRICH 1956; BARBERENA et al. 1970).

Proceso vomeriano: es medial. Se halla cubierto ventralmente por el extremo posterior del vómer. Este proceso se continúa hacia atrás formando la cresta palatina delimitando medialmente el surco palatino que aloja a la rama principal medial del plexo palatino VII. En la base de la cresta y a lo largo de toda su longitud hay una serie de forámenes por donde pasan ramas del nervio palatino a la membrana oral (SKINNER 1973)

\section{Prootico (Fig. 3A)}

Hueso irregularmente trirradiado, forma la parte anterior del segmento occipital y la pared anterior y lateral del cráneo. Dorsalmente articula con el parietal y supraoccipital, posteriormente con el exoccipital y cuadrado, ventralmente con el basiesfenoides y basioccipital.

En la cara medial en la base del proceso alar, está el receso para la vena cerebral media (OELRICH 1956) que desemboca en la muesca del trigémino. Por delante de este receso hay una prominencia que corresponde a la bula timpánica. Anterior y ventral a ésta se encuentra el receso acústico, que aloja al ganglio auditivo anterior y a tres forámenes: dos dorsales que transmiten dos brazos de la rama anterior del nervio acústico y, el tercero, de posición ventral con respecto a los otros dos, para el nervio facial (OELRICH 1956). En la misma cara y anteriormente al proceso posterior se encuentra el cavum capsularis, en cuyo interior está el receso ampular anterior que aloja a las ampollas anterior y externa. Ventral al cavum capsularis se halla el receso lagenar que se continúa con el basioccipital que aloja a la lagena (OELRICH 1956). Presenta tres procesos.

Proceso alar: ancho, su borde anterior forma la crista allaris. Internamente a ella corre el canal semicircular anterior que se abre dorsalmente en la superficie de articulación con el supraoccipital. La crista allaris finaliza en el borde posterior de la muesca del trigémino.

Proceso inferior: su borde dorsal forma la muesca del trigémino. Articula ventralmente con el borde posterior del proceso alar del basiesfenoides y con el basioccipital. Lateralmente lleva la cresta proótica que se extiende hasta el proceso 
posterior. Por debajo de ella, queda delimitado el canal de la vena yugular que aloja a la vena yugular interna, al ganglio facial y al nervio hiomandibular (SKINNER 1973). En este canal se abre también el forámen facial para el nervio facial (OELRICH 1956).

Proceso posterior: articula con los procesos paraoccipitales del exoccipital. Medialmente y en la base del proceso se abre el forámen del canal semicircular lateral.

\section{Región occipital}

\section{Basioccipital (Fig. 2B, 3B)}

Hueso impar, situado medialmente en la parte posterior de la base del neurocráneo formando el piso del mismo. Posteriormente forma el cóndilo occipital. Articula anteriormente con el basiesfenoides, láterodorsalmente con el proótico y pósterodorsalmente con el exoccipital.

La cara dorsal es cóncava, aloja a la médula oblongata. La ventral es plana y presenta lateralmente dos proyecciones muy conspicuas, los tubérculos esfenoccipitales en los cuales finalizan las cristas interfenestralis y tuberalis del exoccipital.

\section{Exoccipital (Fig. 3B)}

Hueso de forma irregular, forma la pared pósterodorsal del neurocráneo, el borde lateral del forámen magnum y la porción lateral del cóndilo occipital. Articula dorsalmente con el supraoccipital, lateralmente con el proótico y ventralmente con el basioccipital.

Lateral y posteriormente lleva los procesos paraoccipitales con tres superficies de articulación para los procesos posteriores del parietal, para el supratemporal y para el cóndilo cefálico del cuadrado. La cara interna de este proceso es rugosa y articula con el proceso posterior del proótico. En la base se abre el forámen del canal semicircular lateral.

En la cara inferior del hueso y en la base de los procesos paraoccipitales se abren dos agujeros separados por la crista interfenestralis, el más dorsal corresponde a la ventana oval (OELRICH 1956; BARBERENA et al. 1970), por debajo de éste se encuentra la ventana redonda, ubicada en una depresión llamada depresión occipital (OELRICH 1956; BARBERENA et al. 1970) circundada por delante por la crista interfenestralis y por detrás por la crista tuberalis. Ambas finalizan a nivel de los tubérculos esfenoccipitales del basioccipital.

En la cara externa entre el cóndilo occipital y el proceso paraoccipital hay tres forámenes: el más dorsal pertenece al rasgado posterior para el nervio vago (OELRICH 1956), ventral a éste y cerca de la superficie condilar se encuentra el agujero para la rama dorsal del hipogloso; ventral al agujero rasgado y cerca de la crista tuberalis está el agujero rotundo para la salida de la rama ventral del nervio hipogloso.

En la cara medial del hueso se encuentra la bula timpánica.

En la cara interna hay una gran concavidad, el cavum capsularis, con tres agujeros: el más dorsal corresponde al vestíbulo, por debajo se encuentra la ampolla posterior, y ventral a ésta, la lagena. 


\section{Supraoccipital (Figs 2A, 3B).}

Hueso impar. Forma el techo de la parte posterior del neurocráneo y el borde dorsal del forámen magnum. Articula anteriormente con el parietal, lateralmente y en toda su extensión con el proceso alar del proótico, pósterolateralmente con el proceso paraoccipital del exoccipital.

Dorsomedialmente corre la cresta occipital (OELRICH 1956). cuyo ápice lleva el proceso mediano cartilaginoso remanente del proceso ascendente del techo sinótico (OELRICH 1956; BARBERENA et al. 1970) que penetra en una fosa situada en el borde posterior del parietal.

Dorsalmente se distinguen los canales semicirculares anteriores y posteriores que se continúan en el proótico y exoccipital respectivamente y cuyas aberturas se encuentran en la superficie de articulación de dichos huesos. Ventralmente en el borde ánterolateral se encuentran los procesos marginales que sirven de fijación a la tenia marginalis. Pósterolateralmente hay dos estructuras que forman parte de las bulas timpánicas y llevan en su interior el forámen endolinfático que transmite el conducto linfático a la cavidad craneal.

\section{Huesos de la mandíbula. Serie labial}

\section{Dentario (Figs 4A,B)}

Hueso alargado, situado en la región más anterior de la mandíbula. Articula dorsal y ventralmente con el esplenial Dorsalmente lleva el surco dental (SKINNER 1973) que sostiene a los dientes subpleurodontes (PRESCH 1974). Debajo de este surco está el canal alveolar inferior por donde pasa el nervio del mismo nombre. Este canal se abre en seis forámenes mentales dispuestos en hilera a lo largo de la línea media de la cara lateral para las ramas cutáneas del nervio alveolar inferior (OELRICH 1956). Por debajo del canal alveolar se encuentra el canal de Meckel, que se abre en la extremidad anterior del hueso.

$\mathrm{Su}$ extremidad posterior proyecta tres ramas: dorsal, media y ventral. La dorsal es ancha, lleva una gran carilla articular en la cual se apoya el proceso basal del coronoides; la media es pequeña, penetra como cuña entre los huesos suprangular y angular; la ventral es aguzada, se apoya sobre una depresión del hueso angular.

\section{Suprangular (Figs 4A,B)}

Hueso grande con el extremo anterior aguzado. Forma parte de la pared lateral del forámen mandibular y el borde dorsal del mismo. La cara lateral lleva dos forámenes, uno situado en la región dorsomedial del hueso, forámen suprangular anterior; el otro, situado próximo a la sutura con el articular, el forámen suprangular posterior. Ambos permiten el pasaje de las ramas cutáneas del nervio alveolar inferior (BARBERENA et al. 1970; SKINNER 1973). Medialmente el hueso está perforado por un gran forámen que transmite ramas del nervio mandibular.

Articula dorsalmente con el proceso posterior del coronoides, anteriormente con el dentario, ventralmente con el angular y posteriormente con el articular.

\section{Angular (Fig. 4B)}

Hueso alargado con los extremos aguzados, situado ventralmente. Forma la pared medial del canal de Meckel. El extremo anterior lleva una carilla en la que se 
apoya el dentario. En la cara interna del hueso se encuentra el forámen para el nervio milohioideo posterior. Articula dorsalmente con el suprangular, anteriormente con el dentario y posteriormente con el articular y ventralmente con el esplenial.

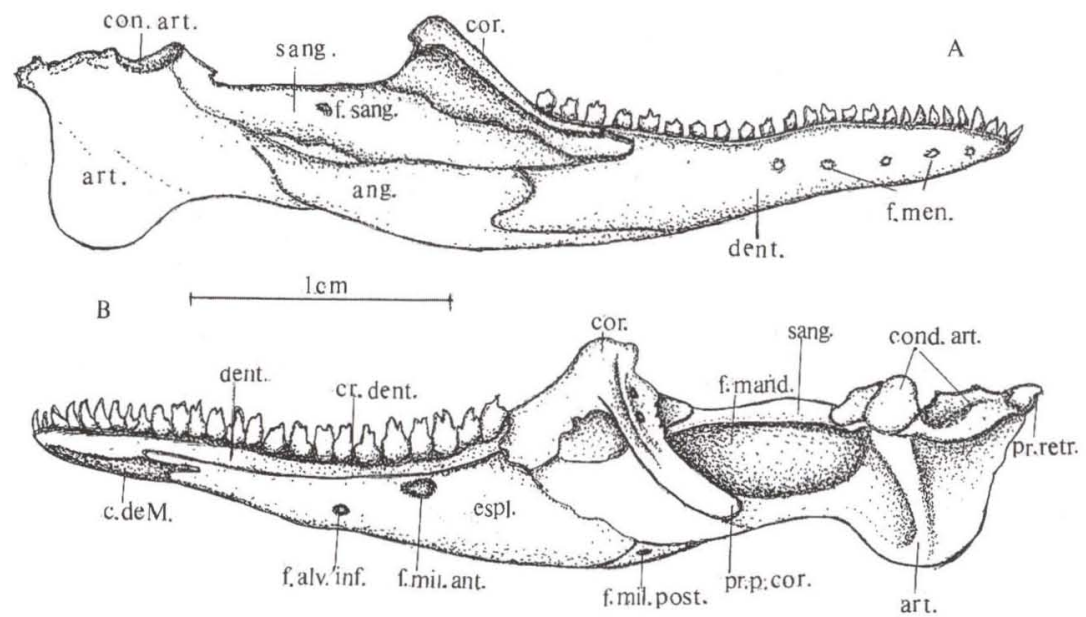

Fig. 4.) Mandibula de $A$. ameiva, (4A) vista labial y (4B) vista lingual. (ang.) Angular, (art.) articular, (c.de M.) canal de Meckel, (con.art.) cóndilo articular, (cond.art.) cóndilo articular, (cor.) coronoides, (cr.dent.) cresta dentaria, (dent.) dentario, (espl.) esplenial, (f.alv.inf.) forámen alveolar inferior, (f.mand.) fóramen mandibular, (f.men.) forámenes mentales, (f.mil. ant.) forámen milohioideo anterior, (f.mil.post.) forámen milohioideo posterior, (f.sang.) forámen suprangular, (pr.p.cor.) proceso posterior del coronoides, (pr.retr.) proceso retroarticular, (sang.) suprangular.

\section{Articular (Figs 4A,B)}

Hueso alargado, aguzado anteriormente, situado en el extremo posterior de la madíbula. Presenta cuatro procesos.

Proceso anterior: alargado, aguzado, forma el piso y el borde medial del forámen mandibular. Articula dorsalmente con el suprangular, anteriormente con el esplenial, y ventralmente con el angular.

Proceso angular: situado ventromedialmente.

Proceso retroarticular: situado por detrás del cóndilo articular, posterior a éste hay una depresión en cuyo interior se encuentra el forámen para la cuerda timpánica y la arteria condilar superior (OELRICH 1956).

Proceso condilar: forma el cóndilo que articula al hueso cuadrado.

\section{Serie lingual}

\section{Esplenial (Fig. 4B)}

Hueso relativamente grande, aplanado, situado en la cara interna de la mandíbula, completa la pared medial del canal de Meckel. Su extremo anterior 
aguzado con una pequeña escotadura delimita el orificio de salida del canal meckeliano. Su extremo posterior penetra como cuña entre los huesos articular y angular.

Articula dorsalmente con el dentario y coronoides, ventralmente con el angular y dentario.

Presenta dos forámenes: uno situado en la parte media y dorsal del hueso que corresponde a la rama lingual del nervio alveolar inferior y a la cuerda timpánica (SKINNER 1973); el otro, más pequeño, corresponde al forámen milohioideo anterior, para el nervio del mismo nombre y para otra rama del nervio alveolar inferior (SKINNER 1973). La cara interna del hueso constituye el canal de Meckel.

\section{Coronoides (Figs 4A,B)}

Hueso de forma aproximadamente triangular y es el más dorsal de la mandíbula. Se distinguen tres procesos.

Proceso dorsal: de ápice redondeado, lateralmente es recorrido por una cresta prominente que se inicia a nivel del ápice y se extiende hacia el tercio posterior del proceso basal anterior.

Proceso basal anterior: consta de dos ramas, una lateral y otra medial. $\mathrm{La}$ lateral es angosta con el extremo anterior biselado. La medial es corta y junto con la rama lateral forma un ángulo en el que penetra el extremo posterior y dorsal del dentario. Articula ventral y anteriormente con el esplenial, posterior y ventralmente con el articular y suprangular.

Proceso basal posterior: alargado, curvado, su cara lateral es recorrida por una cresta que nace en el borde posterior del proceso dorsal y llega a la base del proceso.

Está formado por dos ramas, una dorsal pequeña, articula con el borde dorsal del suprangular y, la otra, dirigida ventralmente, se apoya sobre el articular. Ambas ramas junto con el articular forman el borde anterior del forámen mandibular.

Denticion (Tab. II)

Constituido por dientes subpleurodontes (PRESCH 1974), con curvatura ánteroposterior. Morfológicamente responde a una secuencia de dientes uni, bi y tricuspidales en el premaxilar, maxilar y dentario.

Serie premaxilar: dientes cónicos, unicuspidales, su rango varía entre 7 y 12 .

Serie maxilar: dientes cónicos, unicuspidales entre 1 y 8 , continúan los bicuspidales entre 10 y 19 , luego los tricuspidales entre 2 y 11 . La serie se inicia con dientes curvados en sentido ánteroposterior, en cuanto que los últimos tienen orientación láteromedial.

Serie mandibular: varía entre 24 y 26 dientes. La serie se inicia con dientes unicuspidales entre 1 y 3 , continúan los bicuspidales entre 16 y 21 , por último los tricuspidales ente 3 y 10 .

ro de cúspides.

La fórmula dentaria es: $\frac{10^{1} 3^{1} 12^{2} 3^{3}}{4^{1} 12^{2} 10^{3}}$ donde el exponente representa el númeHioides (Fig. 5)

Elemento cartilaginoso con algunas regiones constituidas por cartílago calcificado. Consiste en un cuerpo cartilaginoso con forma de herradura, el basihial, del cual parte anterior y medialmente una pieza impar, aguzada, el proceso entogloso 
o lingual. Anterior y lateralmente parten dos proyecciones aplanadas dorsoventralmente, los hipohiales. El elemento más lateral del hioides está representado por los ceratohiales, que se dirigen levemente hacia atrás y afuera, articulan con los hipohiales a la altura del basihial. Internamente, respecto a los ceratohiales, parten del basihial un par de proyecciones comprimidas lateralmente, los ceratobranquiales I, de cartílago calcificado con la extremidad distal cartilaginosa dirigida hacia afuera y atrás. En la unión del basihial con el ceratobranquial I, nace un par de cortos procesos muy delgados de naturaleza cartilaginosa, los ceratobranquiales II.

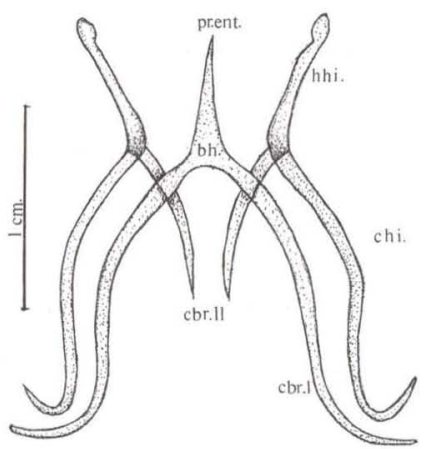

Fig. 5.) Hioides de A. ameiva, vista ventral. (bh.) Basihial, (chi.) ceratohial, (cbr.l.) ceratobranquial I, (cbr.II.) ceratobranquial II, (hhi.) hipohial, (pr.ent.) proceso entogloso.

\section{Aberturas del cráneo}

Abertura exonarina (Figs $2 \mathrm{~A}, 3 \mathrm{~A}$ )

Situada en la región anterior del hocico, de forma ovalada, levemente ensanchada en la región anterior. Está limitada por el premaxilar, maxilar y nasal.

\section{Abertura orbitonasal}

Comunica las fosas nasales con la cavidad orbitaria. Es limitada por el frontal, por el borde medial del proceso palatino del prefrontal y por el borde medial del palatino.

\section{Abertura orbitaria (Fig. 3A)}

Es la más grande del cráneo, tendiendo a una forma oval. Está rodeada por el postfrontal-postorbital, por el proceso frontal del prefrontal, por el frontal, y por el yugal.

\section{Fosa supratemporal (Fig. 2A)}

Situada en el tercio posterior del cráneo. Es limitada por el postfrontal-postorbital, por el parietal, por el postfrontal-postorbital, por el escamoso, y por el proceso supratemporal del escamoso.

\section{Fosa postemporal (Fig. 3B)}

Situada en la región posterior del cráneo. Es ovalada y limitada por el borde ventral del proceso posterior del parietal, por el supratemporal, por el supraoccipital, proótico y proceso paraoccipital del exoccipital. 


\section{Fenestra vomeronasal (Fig. 1B)}

Pequeña, situada en la parte más anterior del paladar. Limitada por el maxilar y por el vómer.

\section{Fenestra exocoanal (Fig. 1B)}

Relativamente grande, de forma aproximadamente triangular, situada detrás de la fenestra vómeronasal. Es limitada por el maxilar, el palatino, y por el vómer.

\section{Abertura interpterigoidea (Fig. 2B)}

Impar, alargada, se ensancha levemente hacia la parte posterior. Es limitada por el borde medial del palatino y pterigoides. El ángulo posterior es formado por el borde anterior del basiesfenoides.

\section{Abertura infraorbitaria (Fig. 2B)}

Pequeña, de forma ovalada, situada en la porción media del piso de la órbita. Es formada por el transverso, el palatino y el pterigoides.

\section{Fosa infratemporal (Fig. 3A)}

Ventralmente incompleta. Está rodeada por el yugal, transverso, pterigoides, postfrontal-postorbital, y por el cuadrado.

\section{CONCLUSIONES Y COMENTARIOS}

Del estudio osteológico del sincráneo de $A$. ameiva se ha inferido la existencia de importantes diferencias morfológicas tanto a niveles intra como interpespecífico. En el primer caso y a través de la aplicación del Test t y del análisis de componentes principales (Fig. 1) han quedado demostradas diferencias altamente significativas $(p<0,01)$ entre machos y hembras en 9 de las 12 variables métricas consideradas. Los caracteres referidos a la longitud, ancho y altura del cráneo (LC, AC y HC), demuestran un claro dimorfismo sexual en el tamaño del cráneo, siendo el de los machos visiblemente mayor que el de las hembras, diferencia que se refleja también en las demás variables, tales como la longitud del hocico (Lho), ancho del premaxilar (Apm), longitud y altura de la órbita (LAO, HAO), distancia entre la narina y la órbita (DNO) y longitud del parietal (LP) (Tab. I).

Asimismo, teniendo en cuenta los caracteres meramente morfológicos de cada hueso en particular se obtuvieron diferencias intersexuales conspicuas en el basiesfenoides, frontal y parietal. En los machos el basiesfenoides es robusto con una crista sellaris prominente y procesos basipterigoides cóncavos; el frontal unido a los nasales a través de una sutura dentada, lleva rugosidades en su cara dorsal, las cuales se acentúan a nivel de la región frontoparietal; el parietal presenta un cuerpo comprimido lateralmente y la abertura interpterigoidea es angosta en toda su longitud.

El hueso pterigoides carece de dientes, carácter éste que difiere de lo observado por PRESCH (1974) para individuos del mismo taxón, pero de los cuales no consigna la procedencia.

A nivel interespecífico, se comparó el cráneo de A. ameiva con otros representantes de la familia Teiidae: Ameiva undulata parva y Cnemidophorus tigris septentrionalis (FISHER \& TANNER 1970); Teius teyou y Teius oculatus (ALVAREZ 
et al. 1987); Cnemidophorus, Dicrodon, Kentropyx y Tupinambis; Cnemidophorus ocellifer y Tupinambis merianae (BARBERENA et al. 1970). Se constató que la estructura robusta del cráneo y la fuerte esculturación en la región frontoparietal es compartido con A. u. parva; Teius teyou y T. oculatus; Cnemidophorus, Dicrodon, Kentropyx y Tupinambis.

La forma de la abertura interpterigoidea es semejante a la descripta para $A$. u. parva, verificándose una notable diferencia con C. tigris septentrionalis; Teius teyou y $T$. oculatus y C. ocellifer que poseen una abertura interpterigoidea triangular con la extremidad posterior ensanchada.

Con respecto a la ubicación del agujero rotundo, en Ameiva ameiva está localizado en el exoccipital, carácter éste que lo comparte con Teius teyou y Teius oculatus; Cnemidophorus ocellifer y Tupinambis merianae, no así con A. undulata parva y $C$. tigris septentrionalis que lo tienen entre los huesos proótico y exoccipital.

Asimismo, la morfología dentaria de Ameiva ameiva responde a una secuencia de dientes uni, bi y tricuspidales, patrón que comparte con otras especies de la familia, tal como lo describe PRESCH (1974) para 22 especies de Ameiva, aludiendo que esta dentición se da en aquellas especies de teidos que poseen preferentemente dieta insectívora. Ligia Krause constató la misma morfología dentaria en Cnemidophorus, Crocodilurus, Callopistes, Kentropyx y Tupinambis, algunos de ellos con dietas omnívora. Por otro lado, Teius teyou y T. oculatus, responden a una secuencia de dientes uni y bicuspidales (Alvarez et al. 1987) al igual que Callopistes maculatus.

La ausencia de dientes en el pterigoides en $A$. ameiva es compartido con $A$. undulata parva (FISHER \& TANNER 1970), carácter que lo diferencia de $A$. bifrontata que posee dientes en el hueso mencionado (PRESCH 1974).

AGRADECIMIENTOS. Al Señor Roberto Hugo Aguirre por la realización de los dibujos definitivos. Al Profesor Eduardo Porcel por la interpretación de los datos estadísticos.

\section{REFERENCIAS BIBLIOGRAFICAS}

Alvarez, B.B.; M.E. Tedesco \& E. Porcel. 1987. Osteología craneal de Teius teyou (Daudin, 1802) (Reptilia, Teiidae). Cuadernos de Herpetología. 3 (2): $1-31$.

Barberena, M.; N. Gomes \& L. SAnchotene. 1970. Osteologia craniana de Tupinambis teguixin. Pub. Esp. Escol. Geol., Porto Alegre, (21): 1-32.

CEI, J.M. 1993. Reptiles del noroeste, nordeste y este de la Argentina. Herpeto-fauna de las Selvas Subtropicales, Puna y Pampas. Mus. reg. Sci. Nat. Torino Monogr. 14: 1-949.

EChternacht, A.C. 1971. Middle American Lizards. Misc. Pub. Mus. Nat. Hist. 55: 1-86.

FisHeR, L. \& W. TANNER. 1970. Osteological and myological comparisons of the head and the thorax regions of Cnemidophorus tigris septentrionalis (Burger) and Ameiva undulata parva

(Barbour y Noble) (Family Teiidae). Brigham Young Univ. Sci. Bull. 11 (1): 1-41. 
Gavrilov, K. 1959. Curso de Anatomía y Fisiología Comparadas. IV:

Esqueleto: (I Parte). Tucumán, Univ. Nac. de Tucumán. Escuela Universitaria de Ciencias Naturales. 106p.

OELRICH, T. 1956. The anatomy of the head of Ctenosaura pectinata (Iguanidae).

Misc. Pub. Mus. Zool. Univ. Michigan 94: 1-122.

PRESCH, W. 1974. A survey of the dentition of the macroteiid lizards (Teiidae, Lacertilia). Herpetologica 30 (4): 344-349 [reprinted].

REESE, A.M. 1923. The osteology of tegu Tupinambis nigropunctatus. Jour. Morphol. 38 (1): 1-16.

SkINNER, M.M. 1973. Ontogeny and adult morphology of the skull of the South African skink Mabuya capensis (Gray). An. Univ. Stellenbosch 48 (3): 1-116. WASSERSUG, R.J. 1976. A procedure for differential staining of catilage and bone in whole formalin fixed vertebrates. Stain Technol. 51: 131-134.

Recebido em 14.XI.1997; aceito em 27.IX.1999. 